\title{
YAP1-NUTM1 Gene Fusion in Porocarcinoma of the External Auditory Canal
}

\author{
Abbas Agaimy ${ }^{1} \oplus \cdot$ Lars Tögel $^{1} \cdot$ Florian Haller $^{1} \cdot$ Johannes Zenk $^{2} \cdot$ Joachim Hornung $^{3} \cdot$ Bruno Märkl $^{4}$
}

Received: 25 April 2020 / Revised: 8 May 2020 / Accepted: 12 May 2020 / Published online: 20 May 2020

(c) The Author(s) 2020

\begin{abstract}
Gene fusions involving the NUTM1 gene (NUT) represent defining genetic markers of a highly aggressive carcinoma type with predilection for the midline structures of children and young adults, hence the original description as NUT midline carcinoma. Recent studies have increasingly documented involvement of the NUTM1 gene in the pathogenesis of other entities as well. We herein describe two cases of auditory canal carcinomas with features of porocarcinoma, both harboring a newly described YAP1-NUTM1 gene fusion. Patients were males aged 28 and 82 years who presented with slowly growing lesions in the external auditory canal. Histologic examination showed monomorphic basaloid and squamoid cells arranged into organoid solid aggregates, nests, ducts, small cysts, and focal pseudocribriform pattern with variable mitotic activity, infiltrative growth, and focal squamous differentiation, particularly in the most superficial part of the tumor. Immunohistochemistry revealed consistent reactivity for CK5, p63 and SOX10 and diffuse aberrant expression of TP53. CK7 expression was limited to a few luminal ductal cells. The androgen receptor and S100 were negative. Next generation sequencing (TruSight RNA fusion panel, Illumina) revealed the same YAP1-NUTM1 gene fusion in both tumors, which was subsequently confirmed by NUT-FISH and the monoclonal anti-NUT antibody. These cases represent a novel contribution to the spectrum of NUT-rearranged head and neck malignancies. This adnexal carcinoma variant should not be confused with the highly lethal NUT carcinoma based on NUT immunoreactivity alone.
\end{abstract}

Keywords NUT carcinoma $\cdot$ NUTM1 $\cdot$ YAP1 $\cdot$ Midline carcinoma $\cdot$ Ear $\cdot$ Head and neck $\cdot$ Auditory canal

\section{Introduction}

Gene rearrangements involving the NUT Midline Carcinoma Family Member 1 (NUTM1) gene (also known as Nuclear Protein in Testis or NUT) mapped to chromosome $15 \mathrm{q} 14$ have emerged as a reliable genetic marker for a highly lethal poorly differentiated carcinoma with strong predilection for mediastinal and sinonasal midline structures, mainly of children and young adults $[1,2]$. With increasing use of NUT

Abbas Agaimy

abbas.agaimy@uk-erlangen.de

1 Institute of Pathology, University Hospital, Erlangen, Germany

2 Department of Otorhinolaryngology, Head and Neck Surgery, University Hospital, Augsburg, Germany

3 Department of Otorhinolaryngology, Head and Neck Surgery, University Hospital, Erlangen, Germany

4 Institute of Pathology and Molecular Diagnostics, University Hospital, Augsburg, Germany immunohistochemistry (IHC) however, NUT carcinoma became more frequently identified over a wide patient's age and in diverse anatomic sites including lateralized (non-midline) organs such as kidney, major salivary glands, extremity soft tissue and others [3-5]. This has led to a wider inclusion of the NUT IHC in the panel used for classifying undifferentiated or unclassified neoplasms irrespective of site. In the vast majority of cases, the recurrent genomic translocation $\mathrm{t}(15 ; 19)$ (q13;p13.1) results in fusion of NUTM1 to the gene Bromodomain Containing 4 (BRD4), a member of the bromodomain gene family in $78 \%$ of cases $[2,6]$. Rarely, other fusion partners have been described, including BRD3 (15\%) as well as non-bromodomain family members like NSD3 $(6 \%)$ and ZNF532 $(<1 \%)[2,6]$.

Almost one half of all NUT carcinoma cases are located in head and neck sites [7]. Sinonasal cavities harbor half of the head and neck cases with the remainder distributed between other organs including the larynx, salivary glands and other exceedingly rare locations [4, 7-9]. We herein describe two cases of auditory canal porocarcinomas 
carrying a YAP1-NUTM1 translocation. Given the young age of one of the patients and hence the risk for misclassification of his tumor as an aggressive NUT carcinoma on the basis of NUT immunoexpression alone, we believe reporting these cases is of utmost relevance for the differential diagnosis of NUT-positive neoplasms in head and neck routine surgical pathology practice.

\section{Materials and Methods}

The two cases were identified prospectively in our routine files. Case one (index case) was initially interpreted as unusual adnexal-type carcinoma on biopsy and due to the young age and unusual site was sent for RNA fusion testing to rule out translocations. This index case was diagnosed shortly before the recent publication by Sekine et al. who described high frequency of NUTM1 rearrangements in poroma and porocarcinoma of the skin [10]. NUT immunohistochemistry (IHC) was performed following the molecular results. Case two was diagnosed as porocarcinoma based on its identity with the index case and NUT IHC was performed directly during routine evaluation. The tissue specimens were fixed in formalin and processed routinely for histopathology. IHC was performed on 3- $\mu \mathrm{m}$ sections cut from paraffin blocks using a fully automated system ("Benchmark XT System", Ventana Medical Systems Inc., 1910 Innovation Park Drive, Tucson, Arizona, USA) and the following antibodies: pankeratin (clone AE1/AE3, 1:40, Zytomed, Berlin, Germany), CK5 (clone XM26, 1: 50, Zytomed), CK7 (OV-TL, 1:1000, Biogenex), p63 (SSI6, 1: 100, DCS), S100 protein (polyclonal, 1:2500, Dako), SOX10 (polyclonal, 1:25, DCS), synaptophysin (clone SY38, 1:50, Dako), (polyclonal rabbit antibody, 1:100, Dako), and anti-NUT antibody (clone C52B1, 1:45, Cell Signaling). Interpretation of results of the NUT IHC was based on published data showing distinctive nuclear immunoreactivity limited to the neoplastic cells. Normal testicular tissue was used as a positive control [3]. As a control, a cohort of eleven other carcinomas of the auditory canal were tested for NUT expression.

Mismatch repair (MMR) deficiency was assessed using immunohistochemical staining for MLH1 (clone ES05, 1:50, Dako), PMS2 (clone EP51, 1:40, Dako), MSH2 (clone G2-19-1129, prediluted, Ventana), and MSH6 (clone MSH6, 1:300, BD Pharmingen). Unequivocal nuclear staining in the tumor cells was considered retained (normal) expression. Normal cells in the background stroma served as internal control.

\section{Next Generation Sequencing and FISH Testing}

Both tumors were tested for gene fusions using the RNA panel. In addition, the index case was also tested for gene mutations using the TST170 DNA gene panel. RNA was isolated from formalin-fixed paraffin embedded (FFPE) tissue sections using RNeasy FFPE Kit of Qiagen (Hilden, Germany) and quantified spectrophotometrically using NanoDrop-1000 (Waltham, United States). Molecular analysis was performed using the TruSight RNA Fusion panel (Illumina, Inc., San Diego, ca., USA) with $500 \mathrm{ng}$ RNA as input according to the manufacturer's protocol. Libraries were sequenced on a MiSeq (Illumina) with $>3$ million reads per case, and sequences were analyzed using the RNA-Seq Alignment workflow, version 2.0.1 (Illumina). The Integrative Genomics Viewer (IGV), version 2.2.13 (Broad Institute, REF) was used for data visualization.

To analyze the mutational status of commonly cancer related genes, DNA was isolated from FFPE tissue sections using the Maxwell 16LEV Blood DNA kit (Promega, Madison, USA) and submitted to hybrid-capture enrichment-based sequencing analysis using the TruSight Tumor 170 (TST170) gene panel (Illumina) according to the manufacturer`s protocol. Libraries were sequenced on a Next Seq550 (Illumina) and analyzed for single nucleotide mutations, insertions, deletions and copy number variations using the TruSight Tumor 170 software (BaseSpace Sequence Hub, Illumina) with human genome hg19 as reference.

Fluorescence in-situ hybridization (FISH) was performed using the ZytoLight SPEC NUT Dual Color Break Apart Probe (ZytoVision, Bremerhaven, Germany) with standard protocols according to the manufacturer's instructions. Fifty tumor cells were visually inspected using a fluorescence microscope. The presence of two pairs of fused green and orange signals was considered normal findings. On the other hand, presence of one fused orange/green signal and one separate orange and green signals indicates translocation.

\section{Results}

\section{Clinical History}

\section{Case 1}

A 29 year-old patient without any significant past medical or family history presented with a slowly growing lesion in the external auditory canal of 24 months duration 
Fig. 1 Representative examples of the appearance of the YAP1NUTM1-rearranged porocarcinoma of the auditory canal on CT (a Case 1) and at surgery (b Case 2). The excised tumor in Case 2 showed a well circumscribed flat-exophytic to warty lesion (c)
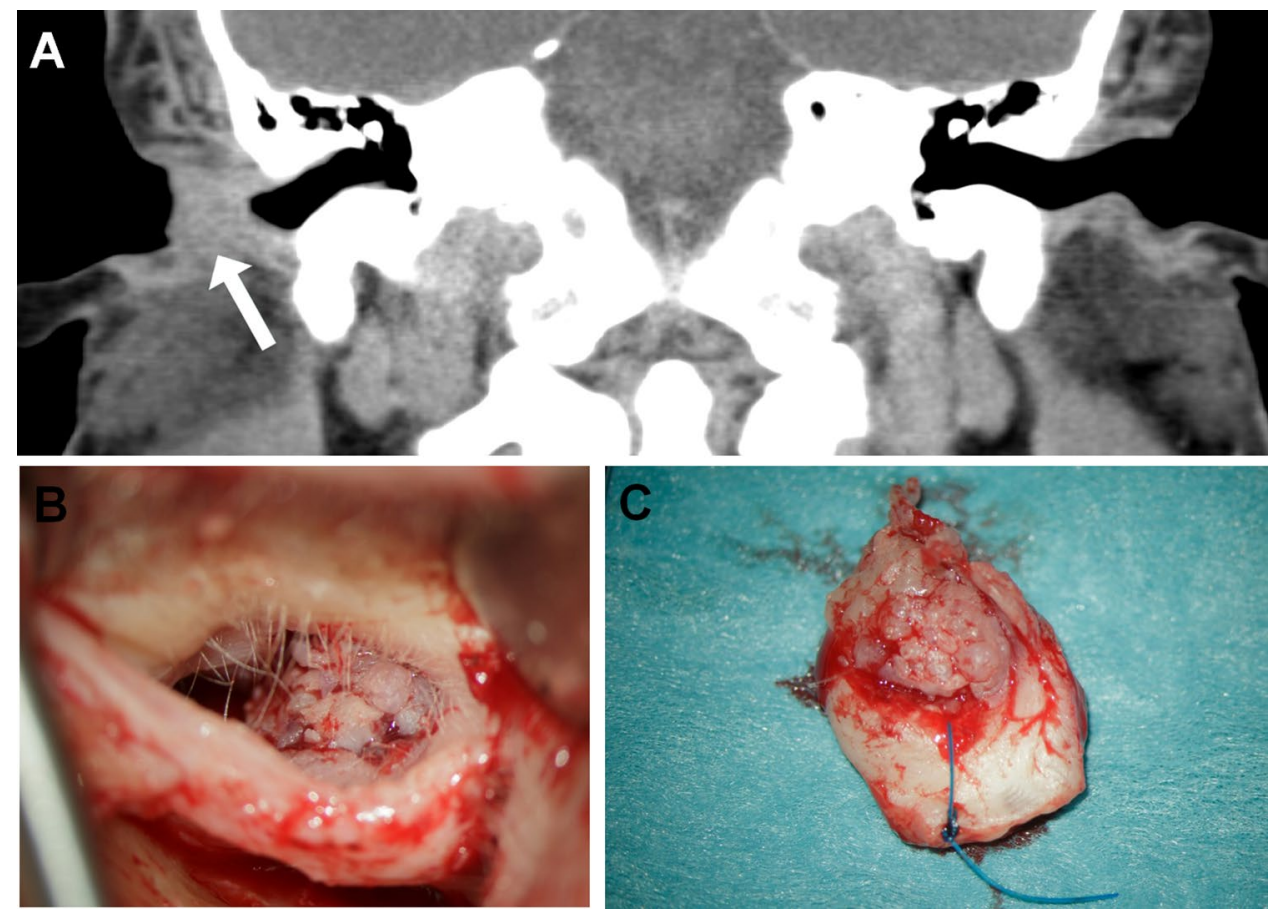

(Fig. 1a). The patient did not attend a first appointment for biopsy, which was obtained 7 months later. There was no evidence of regional lymphadenopathy or distant metastasis. Following complete local excision of the tumor, he received adjuvant radiotherapy. Currently, the patient is alive without recurrence or metastases (12 months).

\section{Case 2}

A 82 year-old man presented with an incidentally found tumor in the right outer ear canal during a routine checkup by his ENT specialist. The patient had no complaints concerning the tumor. In the preoperative CT-scan, a tumor was visible in the outer ear canal with slight bony erosion. There was no sign of regional lymphadenopathy. The tumor was locally excised under the strong suspicion of a carcinoma (Fig. 1b, c). Intraoperative frozen section suggested the diagnosis of porocarcinoma which was then confirmed on permanent sections. No adjuvant treatment was given. The patient is alive and well 6 months after surgery.

\section{Pathological Findings}

Histological features of the two tumors were so strikingly similar that the second case which presented a few months after the first one was interpreted at frozen section as consistent with porocarcinoma. The tumors measured 1.5 and $0.8 \mathrm{~cm}$, respectively. They presented as small polypoid protrusions of the skin with well-defined margins (Fig. 1b, c). Histological examination showed an infiltrating epithelial neoplasm composed of monomorphic atypical mediumsized polygonal to elongated epithelial cells with oval or elongated nuclei, distinctive small nucleoli and a moderate rim of pale-eosinophilic cytoplasm arranged predominantly in well-circumscribed solid nests surrounded by delicate fibrous stroma. Some of the nests showed foci of central necrosis. Other areas showed variable ductal differentiation with prominent microcystic glands with focal pseudocribriforming. The neoplasms merged with a superficial frankly squamous tumor component with higher degree of atypia and associated surface ulceration (Fig. 2). In some areas, the gland-like cystic structures were reminiscent of ceruminal gland lesions or suggestive of a pre-existing benign cystic component. Case two showed prominent involvement of the sweat ducts approaching their superficial openings on top of the epidermis. Here some superficial lateral spreading with replacement of the epidermis to a variable extend was observed. The mitotic activity was low ( $<4$ mitoses/10 high power fields). Foci of abrupt adnexal-type keratinization were seen as well. Representative examples of the two tumors are depicted in Figs. 2 (Case 1) and 3 (Case 2).

Immunohistochemistry showed diffuse expression of pankeratin AE1/AE3, CK5 (Fig. 4a), p63 (Fig. 4b) and SOX10 (Fig. 4c). Diffuse aberrant expression of TP53 was seen in both cases (Fig. 4d). S100, androgen receptor and synaptophysin were negative. CK7 highlighted focal ductal cells. Based on focal antipolar trichilemmal-like nuclear features in Case 1, the mismatch repair proteins MLH1, MSH2, MSH6 and PMS2 were stained; all showed retained physiological nuclear expression (data not shown). The NUT IHC 

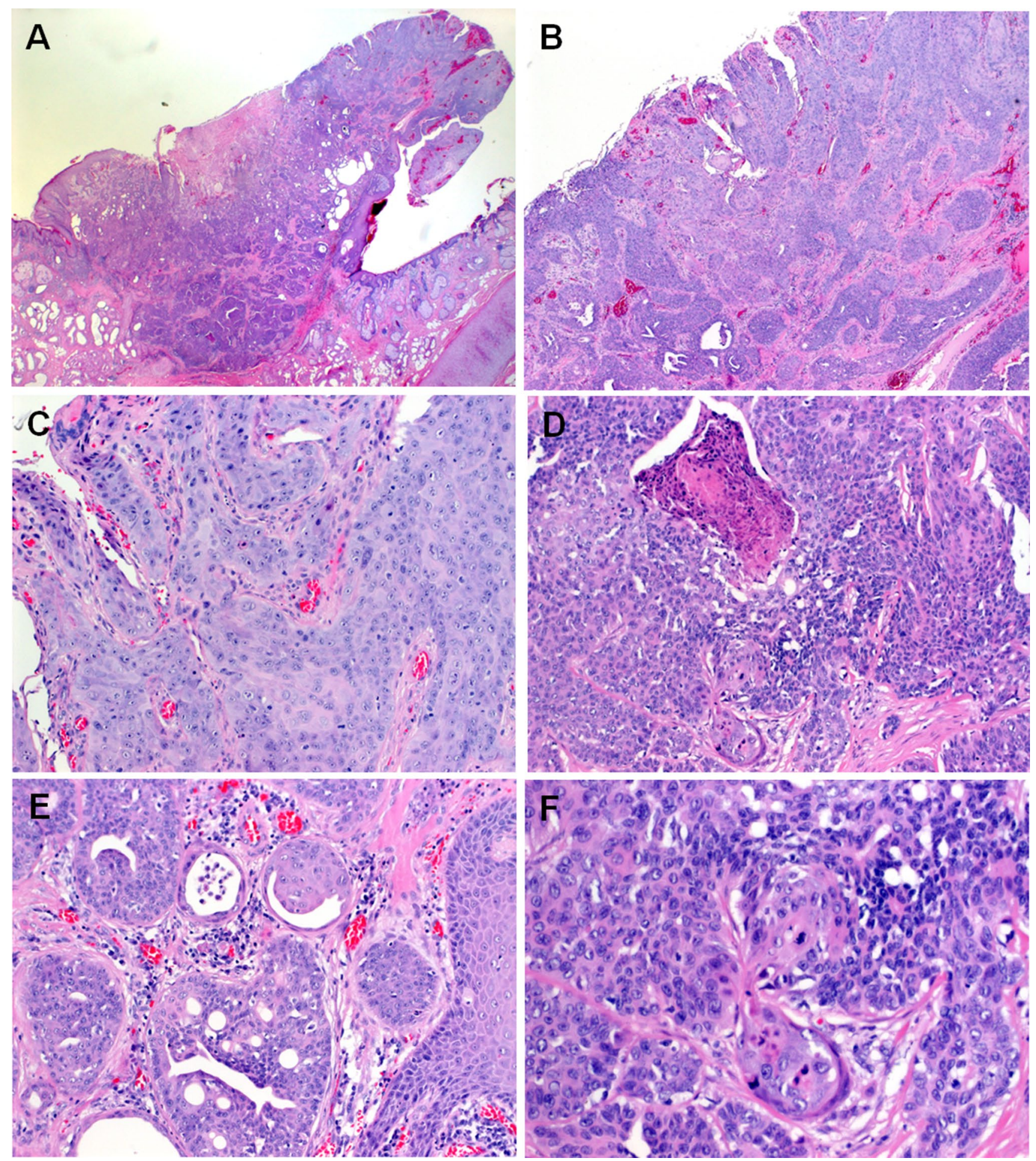

Fig. 2 Representative images of Case 1. a Whole mount section of Case 1. b Closely juxtaposed squamous (upper right) and ductal (lower left) components are seen. c Higher magnification of the squamous component. $\mathbf{d}$ Abrupt keratinization is seen focally. e Prominent

ductal differentiation with pseudocribriforming. f Prominent poroid monomorphic cells interrupted by squamous islands with mitosis (midfield) 

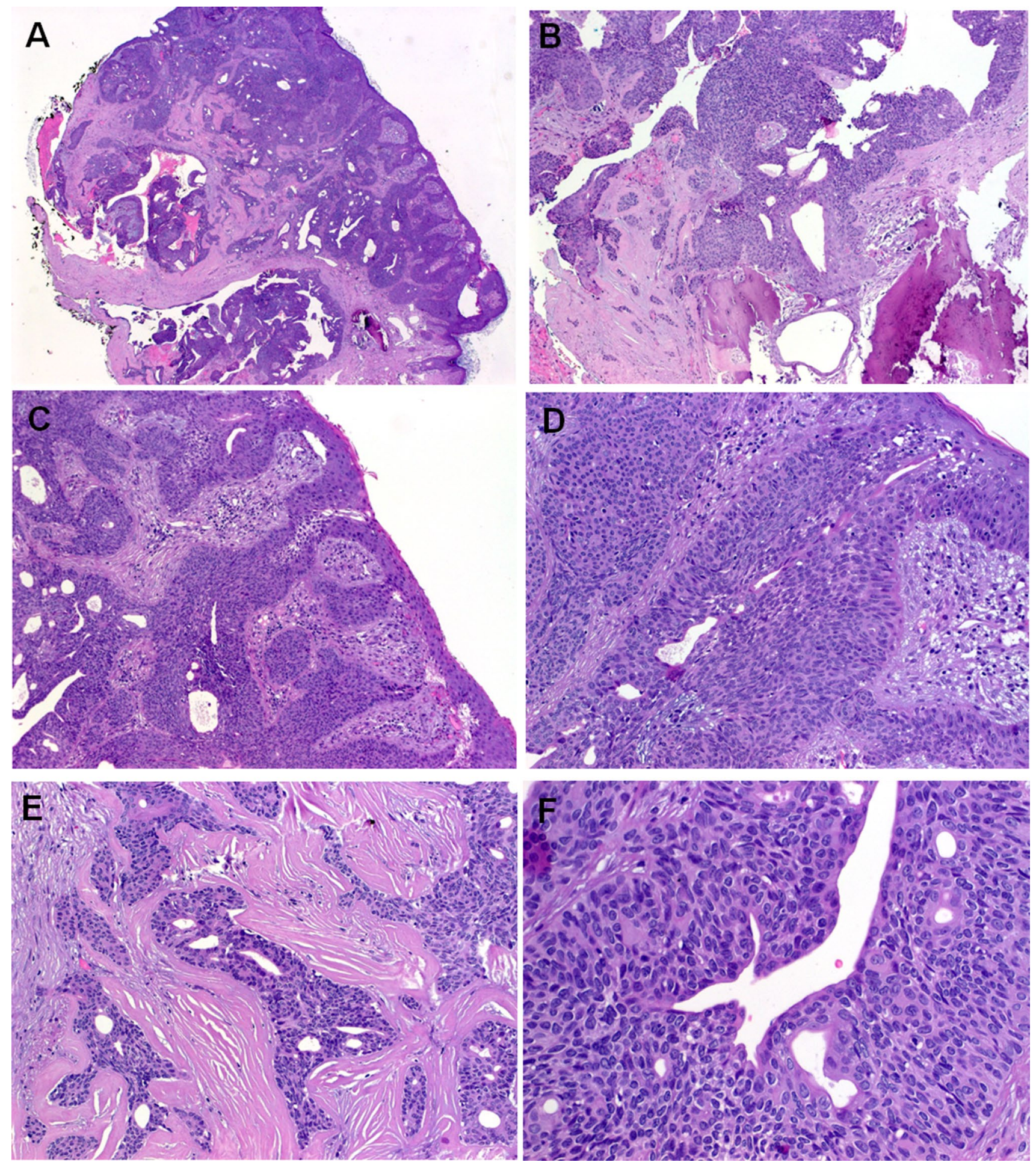

Fig. 3 Representative images of Case 2. a Whole mount shows basaloid neoplasm with prominent ductal and cystic areas. b Focal erosion of underlying bone is seen. $\mathbf{c}$ Prominent replacement of the normal ducts up to the epidermal openings is seen, note lateral spreading

replacing the epidermis to variable extent. d Higher magnification of c. e Prominent sclerosis was seen in purely ductal areas. f Solid aggregates of monomorphic basaloid poroid cells are seen surrounding central ducts 


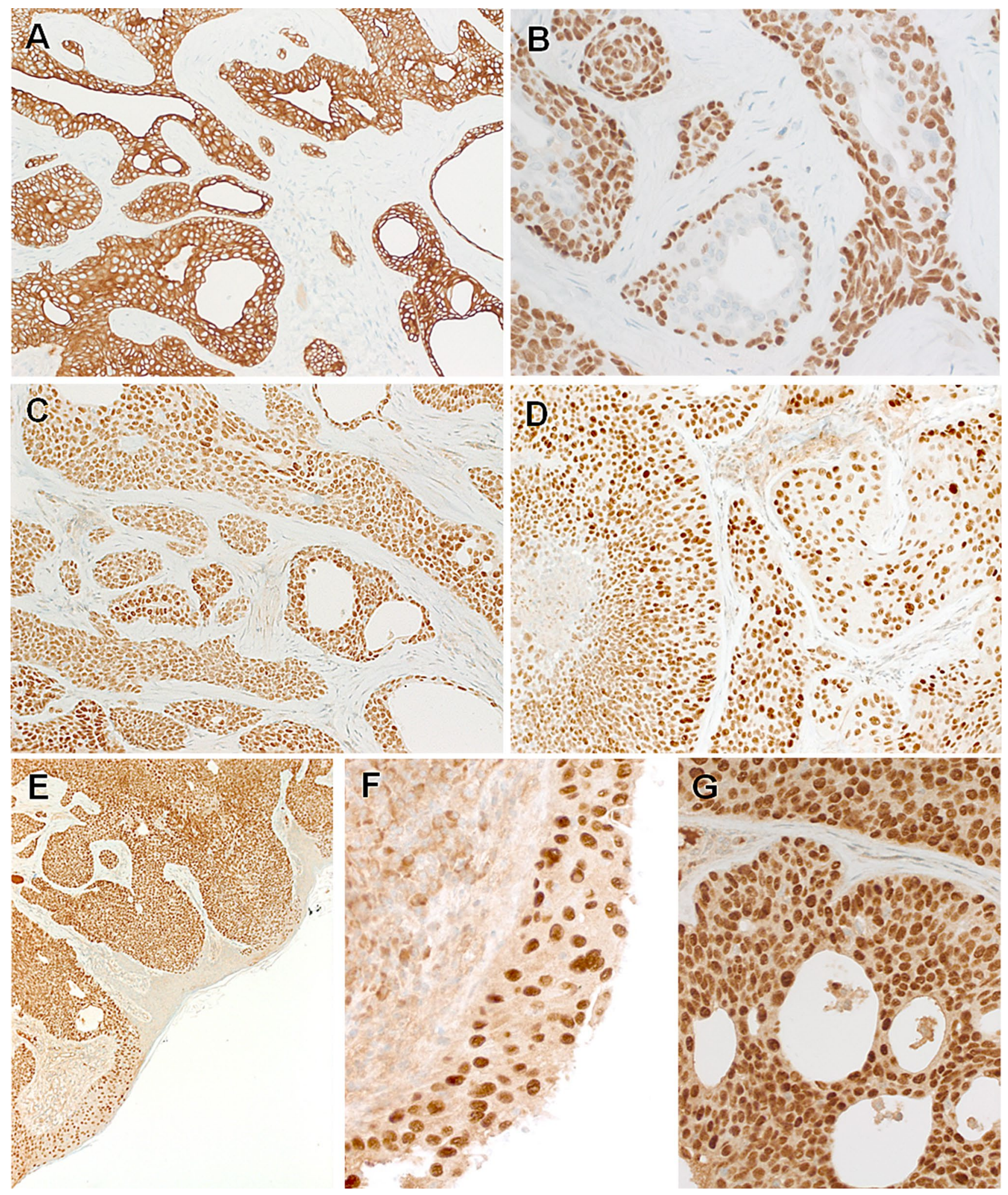

Fig. 4 Representative images of the immunohistochemistry. Both tumors are strongly positive for CK5 (a) and p63 (b), note focal sparing of ductal cells in the p63 stain (b). SOX10 is expressed in all cells of both tumors (c). Aberrant TP53 is observed in both cases (d). Dif- fuse NUT expression is seen and it highlights the lateral spreading along the epidermis sparing non-involved epidermal cells (e, f). At high-power, the NUT reactivity is distinctly nuclear (g) 
showed uniform strong granular punctate to diffuse nuclear staining in all of the neoplastic cells. This staining highlighted the neoplastic cells in areas with reactive-like bland epithelial glands and also stained the frankly squamous superficial component, confirming their neoplastic origin (Fig. $4 \mathrm{e}-\mathrm{g}$ ).

\section{Molecular Findings}

The NGS RNA fusion analysis revealed the YAP1-NUTMI gene fusion in both tumors, in which exons 1-3 of the YAP1 gene were fused in frame to exon 2 of the NUTM1 gene (Fig. 5). FISH testing confirmed the NUTMI gene locus rearrangement in most of the neoplastic cell nuclei (not shown). Furthermore, a mutational screen using a targeted panel of commonly mutated, cancer related genes was performed in Case 1. This analysis revealed no additional mutations, consistent with the role of the YAPI-NUTM1 fusion as sole oncogenic driver mutation in the tumor.

\section{Control Cohort}

The control cohort $(n=11)$ of auditory canal carcinomas of different histological types retrieved from our routine files included six squamous cell carcinomas (SCC; five non-keratinizing and one keratinizing), two adenoid cystic carcinomas, two low-grade adenocarcinomas NOS and one basal cell carcinoma. None of these 11 carcinomas showed NUT expression by immunohistochemistry.

\section{Discussion}

Poroma is a benign skin adnexal neoplasm showing terminal sweat gland duct differentiation [11]. Poromas are composed histologically of a variable combination of poroid cells and so-called cuticular cells. The lesion may be confined to either the dermis or the epidermis, or involve both [11]. Variable ductal differentiation is observed [11]. Porocarcinoma is considered the malignant counterpart of poroma and may arise either de-novo or from pre-existing benign

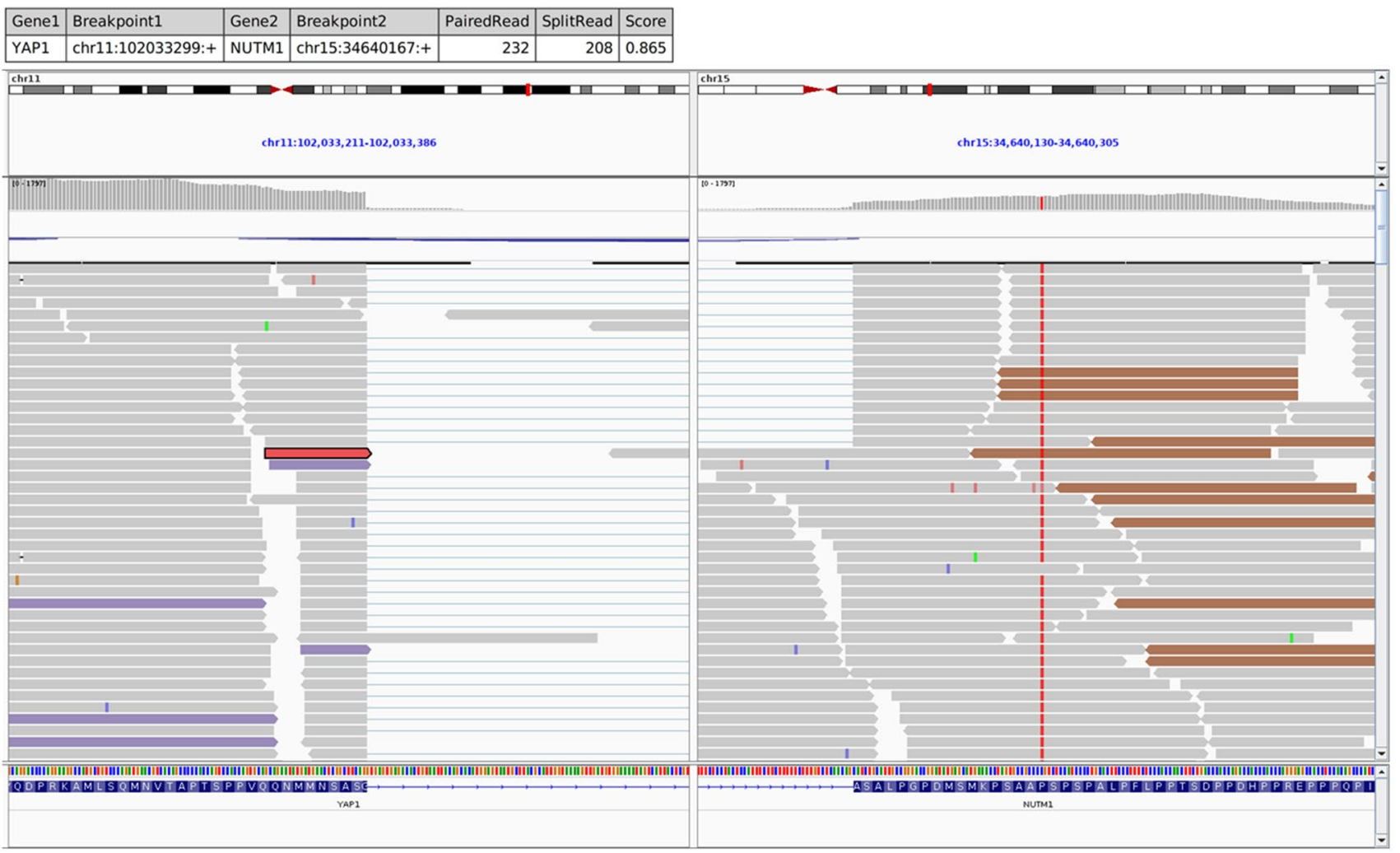

Fig. 5 IGV split-screen view of read alignments of the identified YAP1-NUTM1 Fusion event. Shown are the breakpoints in the YAP1 locus (left) and the NUTM1 locus (right), respectively. Alignments whose mate pairs are mapped to the fusion sequence on the other chromosome are color-coded. The purple colored alignments on the left panel and the brownish alignments on the right are mate-pairs, illustrating the fusion event. All other alignments are colored grey. 
poroma [12]. It typically presents as an ulcerated nodular lesion with a very wide age range (12-91 years) $[12,13]$. The lower extremities are mainly affected, but other sites including the head and neck may be affected as well [12, 13]. Although focal squamous differentiation is considered rare in porocarcinoma, a predominantly squamous variant has been proposed [14]. A small subset of cases is strictly intradermal (so-called porocarcinoma in-situ).

The current cases illustrate examples of porocarcinoma presenting at unusual or unexpected sites. During preparation of this case report, a seminal study reported high frequency of YAP1-NUTM1 gene fusions in subsets of porocarcinoma and poromas [10].

The first case presented herein represents a true challenge due to the unusual age of the patient, unusual presentation and the difficulty and pitfalls associated with interpretation of the molecular results. The poroid nature of this case was not recognized during initial assessment of the preoperative biopsy so that a descriptive diagnosis of adnexal carcinoma was made. As part of our practice to submit unusual and unclassified neoplasms for molecular testing, we sent this case in the setting of initial assessment to our molecular laboratory where the NGS RNA panel revealed the YAPINUTM1 gene fusion. Notably, our diagnosis before and after detection of the NUTM1 gene fusion was concordantly that of an unusual type of malignant skin adnexal neoplasm (adnexal carcinoma) of the auditory canal. This was mainly based on the relatively monomorphic squamoid neoplastic epithelial cells with prominent antipolar nuclear alignment reminiscent of the trichilemmal epithelium. As the study by Sekine et al. [10] was not published at the time of reporting Case 1, we initially considered this unusual genetic alteration of uncertain significance. Accordingly, we emphasized the clear-cut difference between our case and conventional NUT carcinoma in the pathology report to avoid overtreatment $[1,2,6]$. Strong and specific nuclear NUT expression confirmed the genetic finding and is in line with an oncogenically active NUTM1 fusion.

Using RNA sequencing and reverse transcription PCR, Sekine et al. identified high frequency of YAP1-MAML2 and YAP1-NUTM1 fusions in poromas (92/104 cases; $88.5 \%)$ and porocarcinomas (7/11 cases; 63.6\%) [10]. One poroma harbored a WWTRI-NUTM1 gene fusion. YAP1 and WWTR 1 are involved in the regulation of the Hippo signaling pathway via their interactions with TEAD [15]. The tumorigenic effect of YAP1 has been verified in recent studies [15]. Therapeutic opportunities targeting the YAP1/ TEAD-dependent transcription are being tested in different neoplasms [16].

These recent observations underline the need for careful interpretation of immunohistochemical and molecular results in this era of rapidly evolving molecular pathology. Given that the NUT IHC is being increasingly part of the workup of any unusual or unclassified neoplasm, especially by head and neck pathologists, there is a risk to misinterpret such unexpected NUT expression as indicative of NUT carcinoma. Inappropriate diagnosis of NUT carcinoma would be associated with significant impact on patient's treatment and prognosis. Furthermore, detection of NUTM1 rearrangements in benign poromas as well precludes the notion that the NUTM1 gene is a lethal fusion partner in neoplasms and underlines the evolving and continuous role of standard histology and phenotyping in diagnosis and classification of neoplasms.

It is remarkable that both tumors in our study revealed strong and diffuse expression of SOX10, suggesting that SOX10 might be of value in distinguishing porocarcinoma from squamous cell carcinoma on limited biopsies. However, the sparsity of data on SOX10 expression in poroid neoplasms precludes any definitive statement. Cassarino et al. did not detect SOX10 reactivity in 12 tested poromas [17]. However, another study by Lezcano et al. detected SOX10 expression in one of 17 poromas and in one of 4 porocarcinomas [18]. Both studies used same mouse monoclonal anti-SOX10 antibody (clone BC34: Biocare Medical; Concord, California) $[17,18]$ and we used a polyclonal one . Whether this discrepancy is related to the sensitivity and specificity of the antibodies used in different studies or is influenced by the genotype, remains an issue of future studies comparing different antibodies on genetically characterized poroid neoplasms. Indeed, we observed similar strong SOX10 expression in a recent case of YAP1-NUTM1-rearranged benign skin poroma of the upper extremity suggesting that the antibody we use is more sensitive in detecting SOX10 in poroid neoplasms.

In summary, we describe herein the first two cases of auditory canal porocarcinoma harboring a YAP1-NUTM1 gene fusion. Recognition of this entity is mandatory to avoid misinterpretation as lethal NUT carcinoma. These cases add to the expanding spectrum of NUTMI-rerranged neoplasia of the head and neck.

Acknowledgements Open Access funding provided by Projekt DEAL.

Funding No external funding was obtained for this study.

\section{Compliance with Ethical Standards}

Conflict of interest All authors declare that they have no conflict of interest as it relates to this research project.

Ethical Approval All procedures performed in this retrospective data analysis involvinghuman participants were in accordance with the ethical standards of the institutionalreview board.

Open Access This article is licensed under a Creative Commons Attribution 4.0 International License, which permits use, sharing, 
adaptation, distribution and reproduction in any medium or format, as long as you give appropriate credit to the original author(s) and the source, provide a link to the Creative Commons licence, and indicate if changes were made. The images or other third party material in this article are included in the article's Creative Commons licence, unless indicated otherwise in a credit line to the material. If material is not included in the article's Creative Commons licence and your intended use is not permitted by statutory regulation or exceeds the permitted use, you will need to obtain permission directly from the copyright holder. To view a copy of this licence, visit http://creativecommons .org/licenses/by/4.0/.

\section{References}

1. French CA, Kutok JL, Faquin WC, et al. Midline carcinoma of children and young adults with NUT rearrangement. J Clin Oncol. 2004;22:4135-9.

2. French C. NUT midline carcinoma. Nat Rev Cancer. 2014:14:149-50.

3. Haack H, Johnson LA, Fry CJ, Crosby K, Polakiewicz RD, Stelow EB, Hong SM, Schwartz BE, Cameron MJ, Rubin MA, Chang MC, Aster JC, French CA. Diagnosis of NUT midline carcinoma using a NUT-specific monoclonal antibody. Am J Surg Pathol. 2009;33:984-91.

4. Agaimy A, Fonseca I, Martins C, Thway K, Barrette R, Harrington KJ, Hartmann A, French CA, Fisher C. NUT carcinoma of the salivary glands: clinicopathologic and molecular analysis of 3 cases and a survey of NUT expression in salivary gland carcinomas. Am J Surg Pathol. 2018;42:877-84.

5. Stevens TM, Morlote D, Xiu J, Swensen J, Brandwein-Weber M, Miettinen MM, Gatalica Z, Bridge JA. NUTM1-rearranged neoplasia: a multi-institution experience yields novel fusion partners and expands the histologic spectrum. Mod Pathol. 2019;32:764-73

6. Chau NG, Ma C, Danga K, Al-Sayegh H, Nardi V, Barrette R, Lathan CS, DuBois SG, Haddad RI, Shapiro GI, Sallan SE, Dhar A, Nelson JJ, French CA. An anatomical site and genetic-based prognostic model for patients with nuclear protein in testis (NUT) midline carcinoma: analysis of 124 patients. JNCI Cancer Spectr. 2019;4:pkz094.

7. Stelow EB, French CA. Carcinomas of the upper aerodigestive tract with rearrangement of the nuclear protein of the testis (NUT) gene (NUT midline carcinomas). Adv Anat Pathol. 2009;16:92-6.
8. Bishop JA, Westra WH. NUT midline carcinomas of the sinonasal tract. Am J Surg Pathol. 2012;36:1216-21.

9. Hellquist H, French CA, Bishop JA, Coca-Pelaz A, Propst EJ, Paiva Correia A, Ngan BY, Grant R, Cipriani NA, Vokes D, Henrique R, Pardal F, Vizcaino JR, Rinaldo A, Ferlito A. NUT midline carcinoma of the larynx: an international series and review of the literature. Histopathology. 2017;70:861-8.

10. Sekine S, Kiyono T, Ryo E, Ogawa R, Wakai S, Ichikawa H, Suzuki K, Arai S, Tsuta K, Ishida M, Sasajima Y, Goshima N, Yamazaki N, Mori T. Recurrent YAP1-MAML2 and YAP1NUTM1 fusions in poroma and porocarcinoma. J Clin Invest. 2019; 130:3827-32.

11. Sangüeza OP, Cassarino DS, Glusac EJ, Kazakov DV, Requena L, Swanson PE, Vassallo C. Poroma. In: Elder DE, Massi D, Scolyer R, Willemze R, editors. WHO Classification of Skin Tumours. vol. 11, 4th ed. p. 185 .

12. Kazakov DV, Argenyi ZB, Brenn T, Calonje E, Mehregan DA, Mehregan DR, Mehregan DA, Requena L, Sangüeza OP, Santa Cruz DJ, Scolyer RA, Zembowicz A. Porocarcinoma. In: Elder DE, Massi D, Scolyer R, Willemze R, editors. WHO Classification of Skin Tumours. vol. 11, 4th ed. pp. 159-60.

13. Robson A, Greene A, Ansari J, Kim N, Seed B, McKee PT, Calonje PH. Eccrine porocarcinoma (Malignant Eccrine Poroma): a clinicopathologic study of 69 cases. Am J Surg Pathol. 2001;25:710-20.

14. Mahomed F, Blok J, Grayson W. The squamous variant of eccrine porocarcinoma: a clinicopathological study of 21 cases. J Clin Pathol. 2008;61:361-5.

15. Yuan Y, Park J, Feng A, Awasthi P, Wang Z, Chen Q, IglesiasBartolome R. YAP1/TAZ-TEAD transcriptional networks maintain skin homeostasis by regulating cell proliferation and limiting KLF4 activity. Nat Commun. 2020;11:1472.

16. Song S, Li Y, Xu Y, Ma L, Pool Pizzi M, Jin J, Scott AW, Huo L, Wang Y, Lee JH, Bhutani MS, Weston B, Shanbhag ND, Johnson RL, Ajani JA. Targeting Hippo coactivator YAP1 through BET bromodomain inhibition in esophageal adenocarcinoma. Mol Oncol. 2020. https://doi.org/10.1002/1878-0261.

17. Cassarino DS, Su A, Robbins BA, Altree-Tacha D, Ra S. SOX10 immunohistochemistry in sweat ductal/glandular neoplasms. J Cutan Pathol. 2017;44:544-7.

18. Lezcano C, Ho J, Seethala RR. Sox10 and DOG1 expression in primary adnexal tumors of the skin. Am J Dermatopathol. 2017;39:896-902.

Publisher's Note Springer Nature remains neutral with regard to jurisdictional claims in published maps and institutional affiliations. 